THE MAGNETIC PROPERTIES OF HEMATITE. ${ }^{1}$

By T. TOWnsEnd Smith.

THE magnetic properties of crystals have interested many physicists since the early investigations of Plücker and Faraday, and for materials where one deals with paramagnetism or diamagnetism only, the magnetic behavior is dependent in a rather simple way upon the direction that is taken in the crystal. The treatment of this case usually passes under the name of Thomson's Theory, being due fundamentally to Sir William Thomson, though Poisson had previously noted the possibility of a dependence of the magnetic state of a crystal upon the structure of the material.

It may be shown that there are three principal directions in general in a crystal, along which the magnetization and the magnetizing field are similarly directed, and that, in the case treated by Sir William Thomson, ${ }^{2}$ the magnetization in any other direction with a given field may be simply determined from the direction of the field and the susceptibilities along these three directions, using them as the axes in a system of rectangular coördinates. König, ${ }^{3}$ Stenger, ${ }^{4}$ and Tumlirz ${ }^{5}$ attempted to put the theory to experimental test by swinging spheres of calcite and quartz in a uniform field and measuring the period of oscillation, with fair success. There have been also many determinations made of the magnetic constants along the axes of symmetry. A somewhat recent series of determinations of such constants is that of Voigt \& Kinoshita, ${ }^{6}$ in which the forces acting on the specimens in a non-uniform field were used to determine the susceptibility in these directions. There is a rather extended review of such work in Professor Voigt's Lehrbuch der Kristallphysik. ${ }^{7}$

When one turns to crystals in which the field is not proportional to

${ }^{1}$ This work was begun in the Jefferson Physical Laboratory of Harvard University, where almost all of the necessary apparitus was made and where some of the readings were taken. The work is being continued at the University of Kansas.

2 Phil. Mag., March, I85I. Papers on Electrostatics and Magnetism, page 47I.

3 Annalen der Physik, 3r: 273, I887.

${ }_{4}^{4}$ Annalen der Physik, 20: 304, I883; 35: 33I, I888.

5 Annalen der Physik, 27: I33, I886.

${ }^{6}$ Göttingen Nachr., 1907, p. 123. Annalen der Physik, 24: 492, 1907.

${ }^{7}$ Chapter VI., Section 5. 
the resultant intensity of magnetization, one finds that the observations are not numerous and that the phenomena are complex. Professor P. Weiss, ${ }^{1}$ of Zurich, has done more work with such material than anyone else and he, either alone or in collaboration, has published results of measurements made on magnetite and on pyrrhotite (magnetic pyrites). On hematite, which is much less strongly magnetic than either of the two just mentioned, there is some work by Westman ${ }^{2}$ and by Jakob Kunz, ${ }^{3}$ and likewise by Bavink, ${ }^{4}$ who also experimented with tourmalin, garnet, and ilmenite.

The present investigation has to do with hematite, all the tests made being of the component of magnetization parallel to the exciting field. The field strengths used run as high as 3,800 gausses, within which range there is no approach to saturation, either in or perpendicular to the basal plane (plane of symmetry) of the crystal.

The quantity measured in every case was the force which pulled the specimen, a sphere, into the magnetic field, and from this force one may calculate an average intensity of magnetization for the sphere. If the susceptibility is constant throughout a spherical specimen, then to a first approximation the intensity of magnetization calculated from the pull is the value of the intensity at the center of the sphere.

This average value is the value that is given throughout the work below, though in general in hematite the susceptibility will vary, the variation being appreciable in some cases within the range of the fields that exist in the specimen. This is a drawback to the method here used. The non-uniform field is an advantage, however, in that it is possible by its use to test experimentally the homogeneity of the specimen. This may be done by the simple operation of inverting the sphere with reference to the field, in which case the pull will remain unchanged only if the upper and the lower half of the sphere are alike. Within the limits of accuracy of this test, all the specimens showed themselves to be homogeneous.

\section{The Specimens Investigated.}

Hematite crystallizes in rhombohedral crystals, with an axis of threefold symmetry, perpendicular to a plane of symmetry, which is sometimes called the principal plane of the crystal. The crystals are opaque,

${ }^{1}$ Magnetite: C. R., I22: I405, I896; Journal de Physique, Ser. III., 5: 435, I896; L'Eclairage Electrique, 7: 487, I896; 8: 56, 105, 1896; Thesis, Paris, I896; Annalen der Physik, 30: 389, I909 (Quittner). Pyrrhotite: Journal de Physique, Ser. IV., 4: 468, 829, I905.

${ }^{2}$ Upsala Universitets Årsskrift, 1896.

${ }^{3}$ Neues Jahrbuch für Mineralogie, I907, Vol. r, p. 62.

${ }^{4}$ Neues Jahrbuch für Mineralogie, beil. B, I9: 377, I904. 
black in color on the ground faces, but optically uniaxial, according to Foersterling $^{1}$ and others. The crystals are hard and rather heavy, density about 5.25 with good crystals, and contain no water of crystallization.

Crystal clusters are obtained from the Isle of Elba, with beautifully polished faces, which usually give several images when examined with a goniometer by reflected light. In Fig. I are shown several individuals, all of which were obtained from the same cluster and from which the spheres $A_{1}, A_{2}, A_{3}$, and $A_{4}$, referred to below, were cut. The beauty of the faces in a way is deceitful, for, if one of these individuals of a group be cut into, the chances are that the material will be homogeneous for only a short distance back of the faces, being pitted and non-homogeneous a little distance back. The material used in this work was obtained by sawing up the individual crystal units, and grinding until none of the ground faces showed any evidence of cracks or pits, when the piece was assumed to be homogeneous. The results of the tests made on spheres ground from such pieces have shown the specimens so selected to be many of them homogeneous, and a large part of the measurements that I have made have been upon such spheres.

$A_{1}$ was cut purposely from the part of the material back of the clean faces, where the material was pitted and evidently not homogeneous. On test it showed, as was expected, that it was not hematite, having a susceptibility much above that of the other selected specimens and a density likewise far different. The specimens $A_{2}$ and $A_{4}$ are alike, but the specimen $A_{3}$ is not at all like any of the others, showing with a low density a very high ratio between the susceptibility along and perpendicular to the axis ( 0.50 at a field strength of about $\mathrm{I}, 200$ gausses). The most rigorous tests that I have been able to make, however, show no lack of homogeneity. The susceptibility here is also abnormally high and the hysteresis is apparently not great. The density determination was not very accurately made, because of the small size of the sphere and the variations from a true sphere, which are proportionately considerable, so that the lower density referred to above is not to be greatly relied upon.

In addition to the Elba hematite the following specimens have been used in my work:

$$
\begin{aligned}
& F, \text { a twin, from Dognacska, Hungary, } \\
& G \text { from Ouropreto, Brazil, } \\
& J \text { from Schabry, Ural Mountains. }
\end{aligned}
$$

\footnotetext{
${ }^{1}$ Foersterling, Neues Jahrb. f. Mineralogie, beil. B, 25: 344, 1908. Senarmont, Ann. de chim. et de phys. (III.), 20: 445, I847.
} 
In Fig. I are shown photographs of $F$ and $J$. Some of the angles for these two, which were particularly good specimens, were measured on a two-circle goniometer and the angles between the faces were found to be generally within a few minutes of those given by Dana. ${ }^{1}$

For the specimen $F$ all the faces, except the striated " $r$ " (rhombohedral) faces, were large and bright and with reflected light gave clear images, double, with only two or three minutes separating them. The " $r$ " faces were all striated and gave one bright image with an indistinct band, perpendicular to the parallel edges of the faces. The face " $c$ " (basal plane) was slightly pyramidal, rising in regular steps. The crystal was broken off on one edge and the better half is shown, but the lower half exhibited several well-marked faces. The crystal measured just under two centimeters at the widest part.

For the crystal $J$ the striations on the faces " $r$," one of which is here the large irregular face on the left, were rounded, giving a flash of light as the crystal was rotated on the goniometer about an axis in the plane of symmetry and a long streak as it was rotated about a normal to this plane as an axis. An image by reflection was distinguishable, but blurred, and several streaky images from each " $r$ " face were obtained by reflecting sunlight against the wall of the room. These faces were heavily striated, as the figure shows, but there was not the slightest trace of fracture or cracks parallel to these striations nor did the material give such an indication when it was sawn into. Specimens cut from the crystal showed also the magnetic behavior that was to be expected from homogeneous material. The " $c$ " face, the basal plane, just to the right of " $r$," triangular and pyramidal, gave two distinct images in the goniometer 3.5 minutes apart.

The specimen $G$ was a large flat plate, showing fine lines of parting across the two " $c$ " faces, one set parallel to one of the " $r$ " faces and another less distinct set crossing the first at an angle of about sixty degrees. A specimen was cut from this crystal in the part where the lines of parting were finest and least evident, the sawn and ground faces appearing clean and smooth, and the sphere ground from this piece is the one referred to as $G$ below. The faces of these plates were not true enough to give images on reflection, though they were smooth and flat.

The values of the constants for all of the spheres are given below:

\section{The Method of Experimenting.}

An expression for the force exerted upon a small, inductively magnetized sphere $(S)$ in a non-uniform magnetic field has been worked out

${ }^{1}$ Dana, System of Mineralogy. 
Physical Review, Vol. ViII., Second Series. December, r9I6.
Plate I.

To face page 724 .

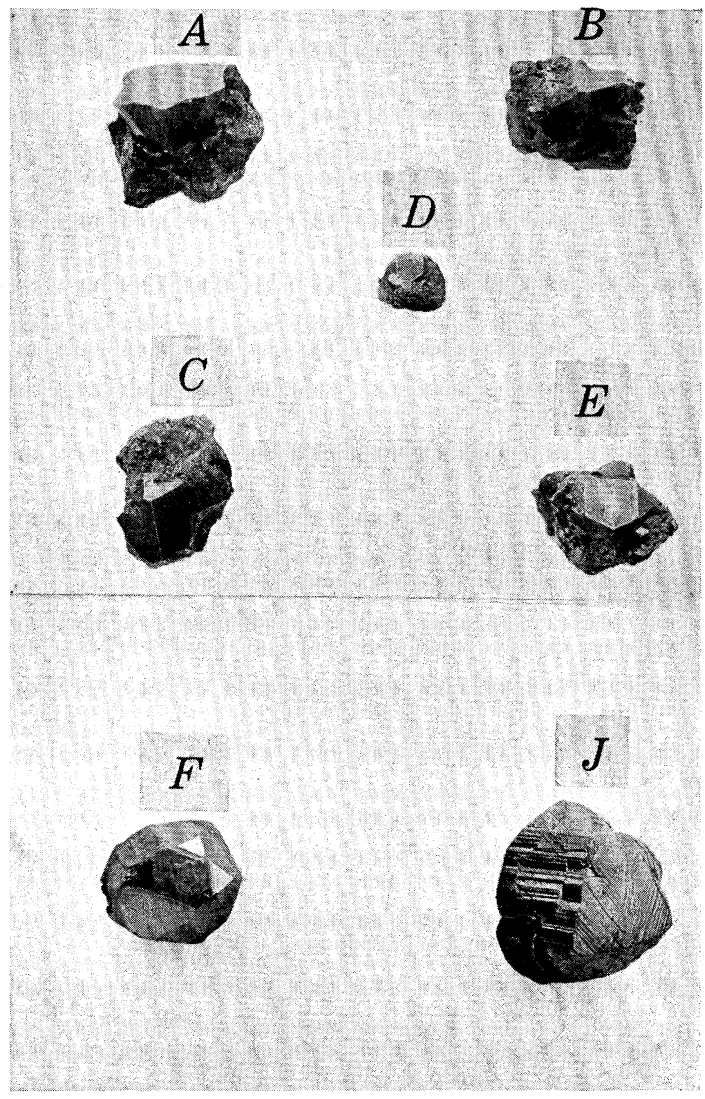

FIG. I. Hematite Crystals. $A-E$, Isle of Elba; $F$, Dognacska, Hungary; $J$, Schabry, Ural Mountains.

T. TOWNSEND SMITH. 
Spheres used in the Magnetic Measurements.

\begin{tabular}{|c|c|c|c|c|c|}
\hline Mark. & Source. & Characteristics. & $\begin{array}{l}\text { Den- } \\
\text { sity. }\end{array}$ & $\begin{array}{c}\text { Diame- } \\
\text { ter. }\end{array}$ & Volume \\
\hline 3 & Elba) & & $(5.22$ & $\begin{array}{c}\mathrm{cm} . \\
0.447\end{array}$ & $\begin{array}{c}\text { c.c. } \\
0.0468\end{array}$ \\
\hline 5 & “ & "Clusters" of single crystals set in an & 5.23 & 0.341 & 0.0208 \\
\hline 9 & & uncrystallized bed. & 5.26 & 1.010 & 0.540 \\
\hline 12 & " & & 5.29 & 0.547 & 0.0857 \\
\hline 10 & “ & & 5.26 & 0.683 & 0.1668 \\
\hline$\left.A_{1}\right)$ & Elba & & 4.90 & 0.581 & 0.1027 \\
\hline$A_{2}$ & “ & & 5.29 & 0.457 & 0.0500 \\
\hline$A_{3}$ & “ & All from the same cluster- $A_{1}$, pitted. & 5.22 & 0.408 & 0.0356 \\
\hline$A_{4} J$ & “ & & 5.27 & 0.367 & 0.0259 \\
\hline$F$ & $\begin{array}{c}\text { Dognacska; } \\
\text { Hungary }\end{array}$ & $\begin{array}{l}\text { Twin, a large well formed crystal-see } \\
\text { Fig. } 1 .\end{array}$ & 5.23 & 0.578 & 0.1011 \\
\hline$G$ & $\begin{array}{l}\text { Ouropreto, } \\
\text { Brazil }\end{array}$ & $\begin{array}{l}\text { Plate with fine striations, forming } \\
\text { lines of parting. }\end{array}$ & 5.23 & 0.593 & 0.1092 \\
\hline$J_{1}$ & Schabry & Fair single crystal, striations on "c" & 5.23 & 0.364 & 0.0253 \\
\hline$J_{2}$ & Urals & and " $r, "$ but no cracks. & 5.23 & 0.345 & 0.0215 \\
\hline
\end{tabular}

by Sir William Thomson, ${ }^{1}$ the work being based directly upon the definitions of the magnetic units. The expression for the force exerted along the $y$-axis has the value

$$
F_{y}=\left[I_{x} \frac{\partial H_{x}}{\partial y}+I_{y} \frac{\partial H_{y}}{\partial y}+I_{z} \frac{\partial H_{z}}{\partial y}\right] v
$$

where $F, I$, and $H$ represent respectively the mechanical force, the intensity of magnetization, and the magnetic field strength, and $v$ is the volume of the element in question.

If one assumes that the magnetization lies in the $x y$-plane the last term will drop out and, if the direction of the $x$-axis be taken as that of the field at the center of the sphere, the second term will have a very small value, so that the final simplified result becomes

$$
F_{y}=I_{x} \frac{\partial H_{x}}{\partial y} v .
$$

The magnet used in most of the work was an electromagnet, with cylindrically shaped pole pieces, hung poles down, and the spheres were placed about level with the lower edge of the pole pieces, as is shown in the upper left-hand sketch of Fig. 2, which is roughly to scale. For adjusting purposes the spheres were cemented to wooden rods by a cement called Cæmentium, which held firmly enough for the work, and which on test proved not to be sufficiently magnetic to cause an observable difference in any of the readings.

1 Papers on Electrostatics and Magnetism, sections 500, 638, 639. 
Cylindrical pole pieces were used because it was found that with such pole pieces the field gradient was very nearly constant over a considerable range, and this is desirable. The pole pieces shown in the sketch have a radius of curvature of about seven centimeters, a depth of a little over three, and a height of about two and a half centimeters, and, where the jaws come closest together at the top, they are separated about seven

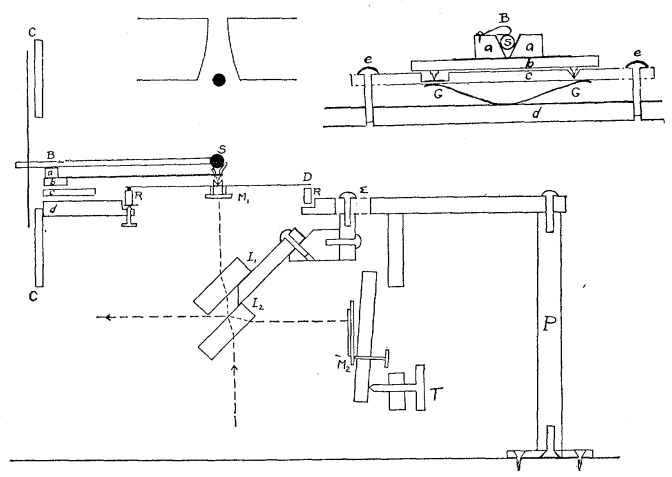

Fig. 2.

The Mounting of the Specimen.

millimeters by a brass plug. The other pole pieces, which were used for lower fields in some cases and with which all the measurements for sphere 9 were taken, are somewhat larger and are separated by a somewhat wider plug.

The field strength was determined by jerking out of the field a narrow exploring coil of thirty turns with an equivalent area of about 6.48 square centimeters. The coil, attached to a light wooden strip, was thrown something like a foot from the point where the field strength was to be determined, and on test the field strength in the neighborhood of the thrown coil was shown to be negligible. The field gradient was determined from the galvanometer deflection obtained by pulling a flat wooden plug from under the exploring coil.

In taking readings of the force a sphere was placed in a little glass cup, which bore upon the center of a three-inch disk of cover glass. The bending of the cover glass, as the field was gradually put on and the weight of the sphere was in part balanced by the force due to the magnetic field, was measured by the shifting of the fringes in a Michelson interferometer arrangement, one mirror of which was attached to the center of the cover glass disk. Fig. 2 shows the arrangement of the interferometer.

$I_{1}$ and $I_{2}$ are the interferometer plates, $M_{1}$ and $M_{2}$ the interferometer 
mirrors. The coarse adjustment of distances is made by moving the plates, the fine adjustment by moving the mirror $M_{2}$, by means of three thumbscrews (only one of which is shown) and a swinging plate, which is controlled by the thumbscrew $T$. There is a flat spring to hold $M_{2}$ against the three thumbscrews on which it bears, and two others hold the swinging plate against $T$.

The somewhat complicated mounting of the disk and specimen appeared to be the simplest way of meeting the experimental requirements, which are that the fringes be steady at all times, that the specimen and mounting be readily removable, and that the specimen be free to rotate so that different directions in the crystal may be in succession made to take the direction of the magnetic field. The disk $D$ is cemented to a glass ring $R R$ at three points, where small glass beads are used to separate the two, and this ring rests upon three thumbscrews in the base plate of the apparatus by a point, groove and plane support. The cement keeps $D$ from slipping, as it otherwise always would, and the whole mounting is readily removable when occasion arises for changing disks. To the disk at its center a small cone-bored jewel bearing is cemented with a trace of cement, and this jewel supports the carriage that bears the sphere under investigation. If the cup, in which the sphere rests, is cemented directly to the disk $D$, any torque that may be exerted upon the sphere makes its presence felt by a twisting and distortion of the fringes.

The construction of the carriage that bears the specimen is shown in the two sections that are given in the figure. The hematite sphere $S$ is cemented to a wooden rod and the two are supported by the glass cup referred to above and the two wooden blocks $a a$. $B$ is a small spring of brush copper that by friction keeps the sphere from turning under the influence of the small couples of the magnetic field. The blocks and the cup are cemented to a strip of cover glass which is in turn supported by three points, of which one rests in the jewel bearing on $D$ and the other two are cemented to the glass strip $b$ and rest upon the glass faced groove and plane cut in the brass plate $c$ above $G G$. The German-silver spring $G G$ holds the plate $c$ up against the adjusting screws $e e$, by which the plate may be raised or lowered, and these screws pass through slots that make possible a right and left adjustment of the plate $c$. These adjustments, together with vertical slots in the plate $C C$, permit the rod $B S$ to be levelled and at the same time to be brought to the center of the divided circle that is marked on $C C$. A glass pointer enables one to read angles on this circle, as the sphere is rotated about the axis $B S$.

The force acting on the sphere in dynes was calculated from the deflec- 
tion produced, measured in fringes. For calibration a light, Germansilver, spiral spring was mounted on a windlass arrangement that could be screwed to the brass bed plate and the windlass on being turned stretched the spring by a known amount. There was no external pull acting on the windlass at the beginning or end of the stretching, except the weight of some twenty centimeters of very light twine, which was used to turn the windlass between fixed stops. The spring was calibrated with light wire weights and a microscope cathetometer. With the disk used in most of the work, a deflection of one fringe would be produced by a force of about twelve dynes.

\section{Preliminary Tests of the Spheres.}

In the earlier work the spheres were stuck on to the wooden rods in a random position with a bit of Cæmentium and then placed on the interferometer, readings of the pull being taken for a number of angles in the zone perpendicular to the rod. The readings were plotted and the maximum determined from the plot, which can be rather accurately done-within probably less than two degrees-if the zone is somewhat removed from the principal plane. Using this maximum for the axis of rotation, a new zone, perpendicular to the first one, is obtained, which should contain the direction of the axis of symmetry of the crystal. This could be tested by resetting with the maximum of this zone for an axis of rotation and getting a third zone, perpendicular to the second, which should likewise contain the axis of symmetry, or the magnet could be rotated a few degrees and thus readings for the neighborhood of the minimum obtained without remounting. Tested in both ways, all of the spheres for which the constants are given in the table above were found to possess a true minimum. Most of the specimens were remounted with this minimum as an axis of rotation, and within the accuracy of the work all the spheres so tested showed no dependence of magnetization upon direction in this zone.

The direction of the maximum may be somewhat more quickly obtained by hanging the sphere, mounted at random, in a magnetic fielda horseshoe magnet is sufficient-and then the direction of the maximum and that of the field should coincide. One obtains the axial zone by using this direction as the axis of a second zone.

\section{The Two Types of Crystals.}

The specimens that I have tested may be divided into two general classes - those which exhibit no signs of hysteresis and show an intensity of magnetization proportional to the field in the direction of the axis of 
symmetry, and another group in which the hysteresis phenomena along the axis are particularly marked. In the first of these two classes, the specimens are paramagnetic along the principal axis and ferromagnetic, that is, exhibit hysteresis, in the principal plane of the crystal; in the second the specimens (all of Elba hematite) are ferromagnetic in all directions, the intensity in the direction of the principal axis being considerably more than for the first group, and the intensity in the principal plane in all observed cases, with the possible exception of $A_{3}$, being noticeably less. There is no difference in the appearance of the specimens, though the density of the first group is apparently a bit lower than is the case with the second. A chemical analysis should be made, but such an analysis would be inconclusive unless made on tested specimens, because spheres cut from crystals from the Island of Elba will be found in both groups. I have specimens from Elba, cut from the beautiful clusters which that district provides; that exhibit magnetic properties which are indistinguishable from those exhibited by a sphere cut from a flat plate of Brazilian hematite $(G)$, and by spheres cut from $J$, which comes from the Ural Mountains and is a specimen very similar in appearance to some of the Elba crystals.

The crystals of the first group are represented by spheres 3, 4, 5, $F, G, J_{1}, J_{2}$ : those of the second group by $9, \mathrm{I} 2, A_{2}, A_{4}$. I have tested a number of other spheres of Elba hematite, most of which would probably belong in group two, though I am somewhat doubtful in several cases as to the homogeneity of the crystals.

In some old tests of Rammelsberg ${ }^{1}$ on Elba hematite, he speaks of two sets of crystals, one with a density of $5.24 \mathrm{I}$ and the other of 5.283 grams per cubic centimeter, which differed only slightly in composition. The heavier specimens contained just under one half of one per cent. of talc earth, and a trace of silicic acid, while the lighter specimens showed traces of both titanic and silicic acids. Both contained a little ferrous iron.

\section{The Crystals Axially Paramagnetic.}

Among the crystals of this group spheres 3 and 5 , from Elba hematite, sphere $G$, from Ouropreto, Brazil, and spheres $J_{1}$ and $J_{2}$, from the Ural Mountains, are almost indistinguishable in their magnetic properties, though the greater size of $G$ makes the measurements on this sphere more reliable than those on the others. The sphere $F$ (twin), from Dognacska, Hungary, shows an intensity well above that of any of the other specimens of this group. All of them exhibit a magnetization parallel to the axis that is proportional to the field within the accuracy

${ }_{1}^{1}$ Annalen der Physik, I04: 528, I858. 
of the measurements made. The magnetization curves for spheres $G$ and $F$ are given in Fig. 3 , both along the axis and in the principal plane.

It was necessary to take the readings in sections with different choices of disks, pole pieces, and magnets for each region of magnetic field strength, because even though the value of the magnetic intensity may

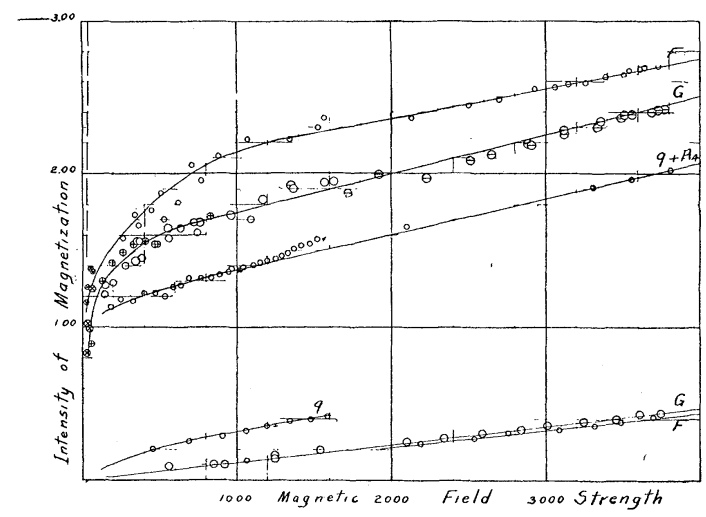

Fig. 3.

Magnetization Curves for Hematite. The upper curves in each case show the intensity of magnetization in the principal plane of the crystal, and the lower curves the intensity along the axis. The broken line indicates the correction that is to be made to the field strength to take into account the demagnetizing action of the sphere.

be changing only slowly, the force exerted upon the specimen and the fringe shift both mount rapidly as the field increases. With a constant susceptibility the force exerted increases nearly in proportion to the square of the field strength, if, as was the case, the field gradient is nearly proportional to the field, and with a hard magnetized body the increase would be in proportion to the field strength, for the force is given by the expression

$$
F=I \cdot G \cdot v .
$$

where $G$ is the field gradient.

All of the readings for fields above about 1,600 gausses were taken with the pole pieces No. I mounted on a large magnet in the laboratory of the physics department of the University of Kansas, while the readings for field strengths below this were made with both sets of pole pieces on a smaller electromagnet with which the majority of my determinations have been made. The readings for values of the field below seventy-five gausses were made with the use of a permanent horseshoe magnet and a Becker balance.

The concordance of the results is not all that could be desired, but considering the variety of circumstances under which the different 
readings were made, I was somewhat surprised to see the different ranges run into one another so well. In Fig. 3 the points obtained for sphere $G$ with the large magnet are shown by the barred circles, the different sets of readings obtained with the smaller electromagnet are differentiated in the plotting, the most reliable probably being the crossed circles. The points for sphere $F$, indicated by smaller circles, represent similarly several distinct sets of readings.

The points determined with the permanent magnet are indicated with an $x$ in the circle. The higher of these readings are probably a bit too high, a correction that was made for a rapidly changing gradient being probably too small.

All of the readings taken in the several runs are shown with the exception of a group of five for the sphere $F$, all of them representing a.fringe movement of I.I fringes and less, and all of them lying appreciably lower than would appear possible from the readings taken with the permanent magnet. These I have thought it legitimate to discard. The values for spheres $3,5, J_{1}$, and $J_{2}$ would lie in good agreement with those for $G$.

The curves represent a case of ferromagnetism with very low intensities, in which there is no sign of approaching saturation at comparatively high fields. ${ }^{1}$ The curves show likewise that the intensity of magnetization must change very rapidly with the field for weak fields. ${ }^{2}$

With the spheres of this first group the amount of hysteresis is relatively small. The forces exerted in non-uniform fields are slightly greater with decreasing than with increasing fields, but the difference is very slight.

For the direction of the axis of symmetry of the crystal the values of the intensity of magnetization are proportional to the field strengths, as is shown by the following figures showing a run for the sphere $G$ :

\begin{tabular}{|c|c|c|c|c|c|c|c|c|c|}
\hline Field & 2,100 & 2,340 & 2,590 & 2,840 & 3,010 & 3,250 & 3,460 & 3,610 & 3,750 \\
\hline Intensity & .247 & .272 & .302 & .328 & .360 & .384 & .398 & .430 & .441 \\
\hline Susceptibility & .118 & .116 & .117 & .115 & .120 & .118 & .115 & .119 & .118 \\
\hline Mean value. & $\ldots \ldots$ & $\ldots$ & $\ldots$ & $\ldots$ & $\ldots$ & $\ldots$ & $\ldots$ & $\ldots \ldots$ & $.117 \times 10^{-3}$ \\
\hline
\end{tabular}

These values are plotted in Fig. 3, together with four others made at lower fields. A similar set of determinations made on the sphere $F$ gave:

\begin{tabular}{l|r|r|r|r|r|r|r}
\hline \hline Field . . . . . . . & 2,190 & 2,540 & 2,760 & 3,090 & 3,320 & 3,490 & 3,700 \\
Intensity . . . . . . & .238 & .271 & .307 & .329 & .357 & .382 & .407 \\
Susceptibility . . . . & .109 & .107 & .111 & .106 & .108 & .109 & .110 \\
\hline Mean value...... & $\ldots \ldots \ldots$ & $\ldots \ldots \ldots$ & $\ldots \ldots \ldots$ & $\ldots \ldots \ldots$ & $\ldots \ldots \ldots$ & $\ldots \ldots \ldots$ & $.109 \times 10^{-3}$ \\
\hline
\end{tabular}

${ }_{1}$ P. Weiss, loc. cit. B. O. Peirce, Am. Jour. Sci., 27: 293, I909.

${ }^{2} \mathrm{~J}$. Westman, loc. cit. 
These values are more accurate than similar figures for spheres 3 and $J_{1}, J_{1}$ showing a result equal to that for $G$, within the experimental error, and 3 giving a result that is apparently a little lower, though again probably within the experimental error of the determinations. In both these latter cases is the calculated intensity nearly proportional to the field strength within the range from about $\mathrm{I}, 800$ to about 4,000 gausses.

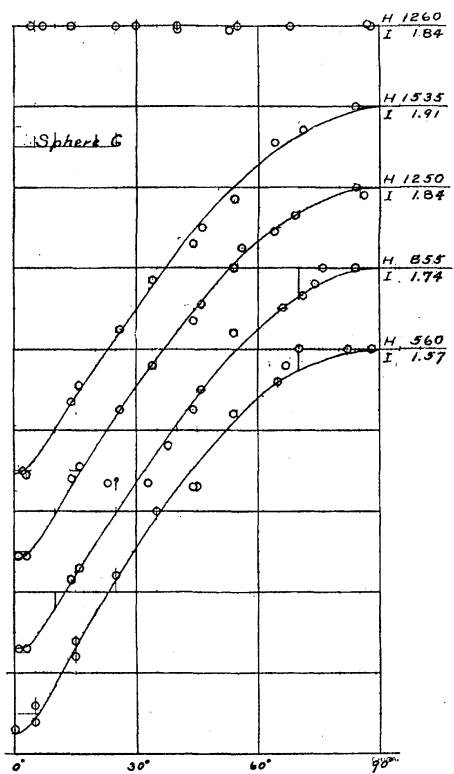

Fig. 4.

Intensity of Magnetization in the Axial Zone for Sphere G. The ordinates give the intensity of magnetization, in terms of the maximum for the zone, the value of which is taken as one. The curves are displaced vertically to avoid confusion. The points above show a zone that is very nearly perpendicular to the axis.
In Fig. 4 are shown plots of the forces exerted upon sphere $G$ for directions which lie between the principal plane and the axis of symmetry and for which the direction of magnetization does not coincide with the field, and similar curves could be given for spheres $F, 3,4$, and 5 . Fig. 4 shows the points for an axial zone for sphere $G$ all plotted in one quadrant, though the readings here represented were taken throughout the whole circumference. The top curve in the figure represents readings taken in the principal plane, nearly, and it is evident that there is no evidence of three-fold symmetry. The constancy here indicated of the component of the intensity parallel to the field has been remarked by Kunz, ${ }^{1}$ using specimens of Vesuvian hematite in the shape of disks with faces parallel to the principal plane.

The set of curves for $G$ shows how slowly the shape of these curves, which represent the intensity parallel to the field, changes with the field strength. Except for a small range near the minimum, one could use the same form for drawing all the curves and get an agreement lying within a probable experimental error, which is relatively larger with weaker fields. The values given for the intensity $I$ are for the principal plane- the intensities in other directions are plotted as fractional parts of the maximum in Fig. 4.

The values plotted to rectangular coördinates in Fig. 4 for a field of I,250 gausses are shown in Fig. 5 plotted to polar coördinates. A plot

1 Loc. cit. 
of this sort, however, does not represent the changes in intensity near the minimum as well as a plot with rectangular coördinates, so that I have used these latter in all the other plots where angles are involved.

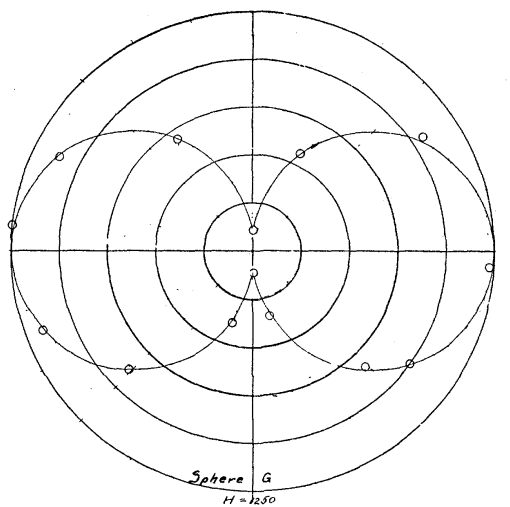

Fig. 5.

The Values of One of the Curves of Fig. 4 Plotted to Polar Coördinates. Field strength I,250 gausses.

\section{The: Axially Ferromagnetic Crystals.}

The curves of Figs. 6 and 7 represent attempts to obtain hysteresis curves for sphere 9, made before I quite appreciated the difficulty that would arise in interpreting the results of the observations. The lower curve of Fig. 6 represents the forces exerted in fields running up to 700

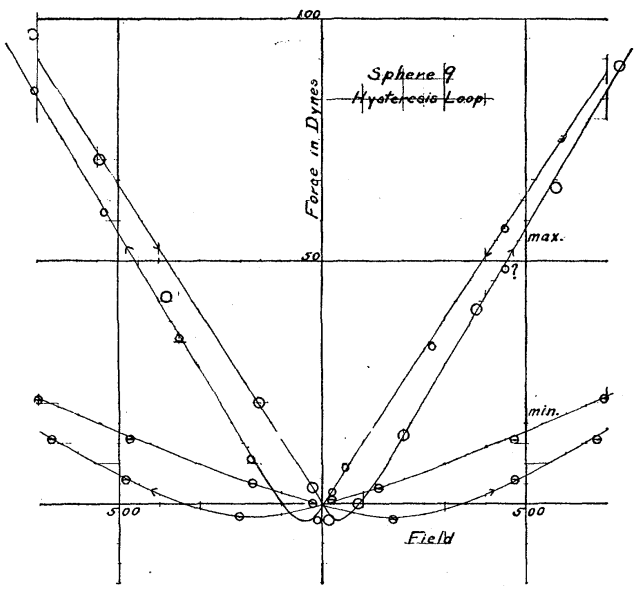

Fig. 6.

The Force Acting on Sphere 9 with Changing Field. Upper curves, direction of the field in the principal plane of the crystal. Lower curves, direction of the field parallel to the axis of the crystal. The curves would close if produced to the maximum field strength, 1,500 gausses. 
gausses upon the sphere when the axis was directed along the direction of the magnetic field and the field was gradually run through a complete cycle, starting from a value near the maximum. The curves, produced, are closed curves at the maximum fields used, about I,500. The values are corrected slightly for a slow fringe shift, due probably to slow temperature changes in the room, which does not affect the evidence of hysteresis, for this lies in the difference between the two curves for increasing and for decreasing fields. The similar curve for the direction of the principal plane is shown also in Fig. 6, drawn to the same scale as

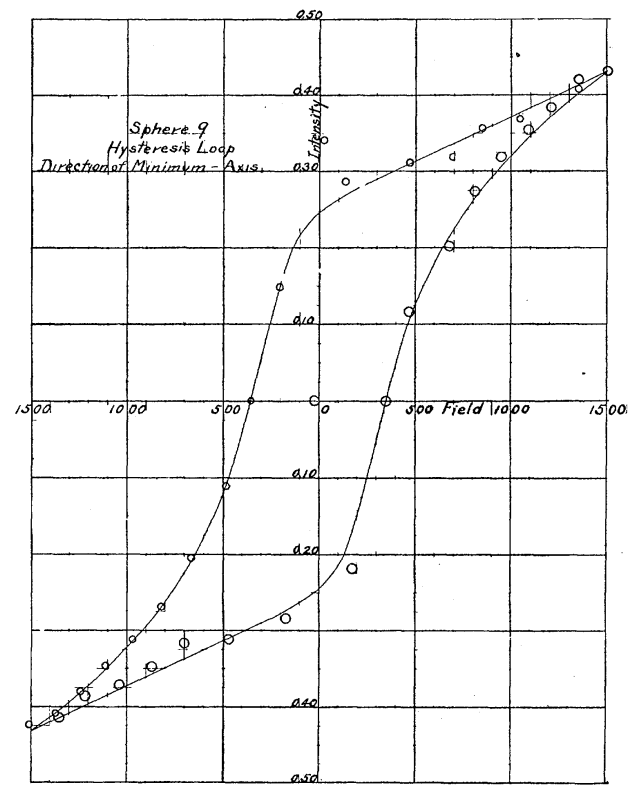

Fig. 7.

Hysteresis Loop for Sphere 9 (Elba). The direction of the field is parallel to the axis of the crystal, and the values of the intensity are calculated from the forces, which are shown in the lower curves of Fig. 6.

for the direction of the axis. Here as before the hysteresis is in evidence, but the difference between the ascending and the descending limbs is less marked.

Both of these curves involve, of course, the field gradient, and I have calculated and plotted the values of the intensity, which are shown for the minimum in Fig. 7. The values on the descending arm for low field strengths are very uncertain. For the maximum direction the curve of the intensity would be similar, though not so pronounced in character. For this direction the readings that I have are not accurate enough to bear translation into an $H I$ curve. 
The high coercive force shown in the figure for the direction of minimum magnetization was otherwise made evident when I hung the sphere 9 by a silk thread between the jaws of a permanent magnet where the magnetic field has a value of about seventy-six gausses, with the axis of suspension in the principal plane. The sphere should normally point, of course, with the plane of symmetry parallel to the magnetic field, because this is the direction of maximum susceptibility. However, if the sphere has been exposed previously to a stronger field, the residual magnetization along the axis might be sufficient to deflect the sphere so that the positions of equilibrium would make an obtuse angle with one another, and this is just what I observed on suspending the sphere. No rotation in the weaker field sufficed to prevent this deviation, though the application of a stronger field changed the sign of the deviation.

Further, the hysteresis in the principal plane was shown by the fact that the sphere might be rotated some $110^{\circ}$ to $120^{\circ}$ from the position of equilibrium and still return through the obtuse angle, and the fact that, when hung with the axis of the crystal vertical, the sphere when rotated would drop back some $20^{\circ}$ to $30^{\circ}$, though if this were allowed for, it might be made to point in any desired direction.

For the two principal directions in the crystal, that is, along and perpendicular to the axis, I have obtained the magnetization curves which are shown in Fig. 3. The points up to field strengths of about $\mathrm{I}, 500$ were obtained with sphere 9. The values at higher fields are for sphere $A_{4}$, for sphere 9 was too large to be introduced into the narrower jaws of the pole pieces (No. I) used at these higher fields. My only dependable value for the direction of the minimum in $A_{4}$ is indicated by a cross ( + ). I have not as yet obtained values for $I$ for crystals of this type below the field strengths here shown, for the strong hysteresis complicates the problem of these determinations.

For intermediate positions readings which exhibit the symmetry of the crystals are plotted in Fig. 8, which shows the component of the intensity parallel to the field for different field strengths in terms of fractional parts of the maximum intensity. The open and the dashed circles represent opposite halves of the zone and the two are so superposed as if the points were plotted for the whole 360 degrees on transparent paper and the plot were then folded over at the maximum. The curves I, 2, and 3 are for sphere 9 , in which the axis is indicated by $90^{\circ}$ and the principal plane by $0^{\circ}$ and $180^{\circ}$. The axis is very nearly in the zone of the plot, as is shown by the curve $3 a$, which is obtained by rotating the magnet through small angles in a horizontal circle, when the specimen is set for the minimum of the zone plotted in the curves. Curve 5 is for 
a zone that is nearly, but not quite, perpendicular to the axis, and indicates that the sphere is isotropic in this plane, within the accuracy of the measurements here plotted. The curve marked 4 is for spheres $\left(A_{2}\right.$ and

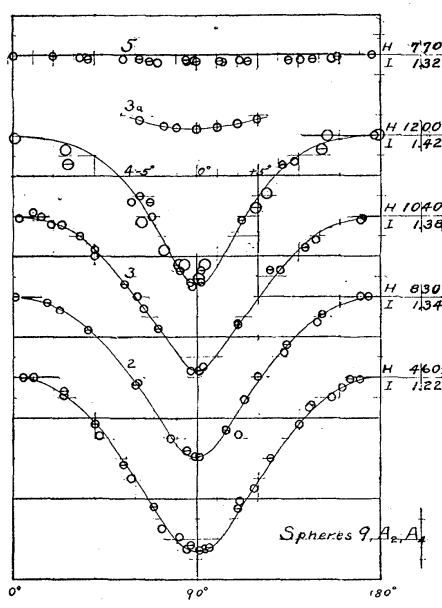

Fig. 8.

Tests on Elba Hematite Spheres (Ferromagnetic). Curves 1, 2, 3, sphere 9, axial zone. Curve 4, spheres $A_{2}$ and $A_{4}$, nearly axial zones. Curve 5 , sphere 9 , zone nearly perpendicular to the axis. Curve $3 a$, sphere 9 , zone perpendicular to 1,2 , and 3 , to a magnified scale showing test for minimum. The ordinates give the intensity of magnetization, in terms of the maximum for the zone, which is taken as one. The curves are displaced vertically to avoid confusion.
$A_{4}$ ), which exhibit properties similar to sphere 9 , at a field strength a little higher than the others. The values for $A_{4}$, large circles in curve 4 , are for a zone that does not quite contain the axis.

Curves 1,2 , and 3 , in which the fringe shifts for the direction of maximum intensity are respectively 5.25 fringes, I I . 75 fringes, and 15.6 fringes exhibit not only a successive increase in the relative value of the minimum intensity, but also a gradual slight change in the character of the curve. The maximum is less sharp for the higher field strengths.

Each of the points on these curves represent the mean of at least two readings with the field in opposite directions, and between readings the specimen was demagnetized by reversals in order to eliminate as much as possible the effect of hysteresis. This was difficult to accomplish at times, both for the direction of the minimum and for oblique directions fairly close to that, the hysteresis being so troublesome in the direction of the minimum that at times it seemed almost impossible to eliminate its influence and get concordant readings. In practically every case persistence and care in demagnetizing by reversals finally did demagnetize the specimen, until the same readings could be obtained by reversing the field.

\section{SuMmary.}

The results of this investigation upon the magnetic properties of hematite may be briefly stated as follows:

I. The symmetry of hematite is magnetically that of an axis of symmetry, with a plane perpendicular thereto in which the magnetic susceptibility is independent of the orientation. ${ }^{1}$

2. In this principal plane hematite is ferromagnetic and exhibits

1 Consult, however, Kunz, loc. cit., pp. 73, 82. 
hysteresis. The magnetic intensity is low and does not approach saturation for fields as high as 4,00o gausses.

3. Parallel to the axis the specimens from many different localities are paramagnetic, but some specimens from Elba exhibit strong hysteresis, as Westman also found, working at low fields with Kragero hematite. $^{1}$ In all cases within the range of field strength available, the intensity of magnetization along the axis is much less than in the principal plane.

4. For intermediate directions, the direction of magnetization does not coincide with the field, and in certain positions the couple exerted upon the material is relatively large. The component parallel to the field has maxima and minima at angles of ninety degrees, as Weiss found for magnetic pyrites. ${ }^{2}$ I have not yet measured the component perpendicular to the field.

It should be noted that the values for the intensity of magnetization here found are in all cases less than those found by Westman and by Kunz. Westman, using a magnetometer method and small rods of hematite, has obtained hysteresis loops with a maximum field strength of about seventy gausses. The ascending and descending limbs of these curves are almost identical above thirty gausses, and for this range his values are about twice what one would expect from an extrapolation of the magnetization curve for the principal plane of sphere 9. Except for the lack of agreement in the values of the intensity, the specimens of Westman are apparently of much the same nature as is sphere 9 above, in that they show hysteresis in an increasing amount as the direction of the field gets closer to the magnetic axis of the crystal. The accuracy of his work did not permit the plotting of hysteresis loops for the direction of the axis.

Kunz ${ }^{3}$ who made use of over roo different individual crystals from different localities, says that the induced current, which was set up in a stationary surrounding coil when a crystal was jerked out of a magnetic field, indicated that every specimen he tried was paramagnetic for field strengths as high as 600 gausses. However, the constants he gives vary widely, in the ratio of fifty to one, and it is evident from the work above that such variations would not be called forth by any lack of accuracy in setting the specimen. The smallest of his constants is almost twice the value obtained in the work above for sphere $G$, and the observed susceptibility, taking the smaller values, very nearly agrees with the value for sphere 9 at 600 gausses, though this latter shows strong hysteresis at higher fields.

1 Loc. cit.

${ }^{2}$ Weiss, Journal de physique, 4: 469, I905.

${ }^{3}$ Loc. cit. 


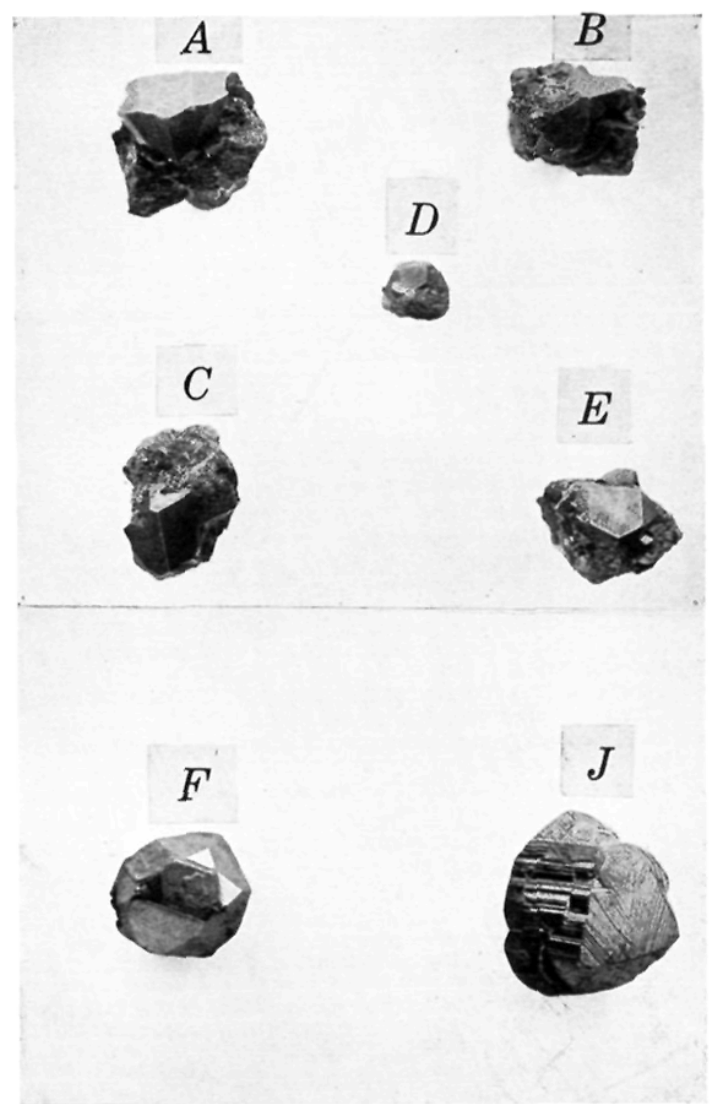

Fıg. I. Hematite Crystals. $A-E$, Isle of Elba; $F$, Dognacska, Hungary; $J$, Schabry, Ural Mountains. 